\title{
Comunicación

\section{Hacia una teoría integrada de la comunicación-mediación en organizaciones de conocimiento de la cibersociedad}

\author{
MigDALIA PINEDA DE ALCÁZAR \\ Y JOHANN PIRELA MORILLO ${ }^{1}$
}

Se presenta un avance de investigación que recoge las reflexiones y las aproximaciones teórico-metodológicas que fundamentan la construcción de una línea teórica integrada sobre los procesos de comunicación-mediación en las organizaciones de conocimiento a la luz del surgimiento de la cibersociedad. Asimismo, se aplica un análisis de contenido de documentos internacionales elaborados por expertos de UNESCO y la Federación Internacional de Asociaciones de Bibliotecas y Bibliotecarios (IFLA).

Palabras Clave: comunicación-mediación, organizaciones de conocimiento, cibersociedad.
The article presents an investigation advance that picks up the reflections and theoretical-methodological approaches that base the construction of a theoretical line integrated on the communication-mediation processes in the organizations of knowledge by the light of the emergence of a cybersociety. The study is supplemented with the analysis of content of international documents elaborated by experts of UNESCO and the International Federation of Associations of Libraries and Librarians (IFLA).

KEY WORDS: communication-mediation, organizations of knowledge, cybersociety.

1 Doctorado en Ciencias Humanas, Facultad de Humanidades y Educación, Universidad del Zulia, Maracaibo-Venezuela

Correos electrónicos: alcazar@iamnet.com y jpirela@luz.ve. 


\section{INTRODUCCIÓN}

Este artículo forma parte de un proyecto de investigación más amplio, ${ }^{2}$ cuyo objetivo general es proponer un "sistema conceptual explicativo" que dé cuenta de los cambios conceptuales que deben introducirse en las organizaciones de conocimiento (bibliotecas, archivos y centros de documentación e información), debido al surgimiento de una cibersociedad y cibercultura, que obliga a replantear los enfoques tradicionalmente utilizados para explicar el proceso de comunicación-mediación en dichas organizaciones.

Las reflexiones y aproximaciones expresadas en el artículo constituyen también los avances de la tesis doctoral: La comunicación-mediación en las organizaciones de conocimiento de la cibersociedad, adscrita a la línea de investigación: comunicación, educación, información y nuevas tecnologías del doctorado en ciencias humanas, de la Universidad del Zulia.

Se formulan planteamientos que conciben la comunicación como un fenómeno que trasciende los ámbitos meramente periodísticos y articula, cual coordenada, los sistemas formales y no formales de mediación del conocimiento; de manera que se asume el carácter complejo y las nuevas racionalidades planteadas por la comunicación telemática e interactiva, que debe guiar la acción de las denominadas organizaciones de conocimiento, instituciones comprometidas con el aprendizaje tecnológico-informativo y la educación "para toda la vida" de los ciudadanos.

Los planteamientos realizados se apoyan en algunos enfoques conceptuales de las ciencias de la información, específicamente las perspectivas bibliotecológica, documentalista e informacionista; de las ciencias de la comunicación, desde donde se caracterizan las mediaciones más allá de los medios de comunicación de masas; y de las ciencias cognoscitivas, que aportan una sólida base explicativa acerca de los procesos cognoscitivos y de aprendizaje que tienen lugar en el sujeto y se hacen más complejos al apoyarse en tecnologías de información y comunicación.

2 "La comunicación-mediación en organizaciones de conocimiento de la cibersociedad". Proyecto registrado ante el Consejo de Desarrollo Científico y Humanístico (CONDES) y el Doctorado en Ciencias Humanas. Universidad del Zulia. 
Además de la revisión, análisis e integración de enfoques, el estudio se complementa con la detección de tendencias proyectadas por expertos de la Organización de las Naciones Unidas para la Educación, la Ciencia y la Cultura (UNESCO) y la Federación Internacional de Asociaciones de Bibliotecas y Bibliotecarios (IFLA). Las conclusiones del análisis teórico y de las tendencias plantean la necesidad de construir una nueva línea teórica-explicativa más acorde con las características de una sociedad que se levanta sobre lógicas diferentes de la producción, acceso y comunicación del conocimiento.

Se considera que los resultados de este estudio pueden aportar importantes elementos de juicio para la formulación de nuevas propuestas curriculares orientadas hacia la formación de profesionales responsables de la comunicación del conocimiento en la cibersociedad, asunto de singular importancia que toca incluso la renovación epistemológica y conceptual de las carreras de bibliotecología, archivología, documentación y ciencia de la información, frente a los desafíos de una nueva sociedad. Igualmente, se busca aportar referentes conceptuales en el diseño y puesta en marcha de políticas y programas para las organizaciones de conocimiento, adaptados a las nuevas condiciones y características que emergen en la actual sociedad.

\section{MetodoloGía}

El artículo es el producto de una investigación teórica y documental que partió de la construcción conceptual del objeto de estudio y su caracterización, e incluyó una revisión y análisis de enfoques conceptuales sobre la comunicación-mediación del conocimiento, haciendo énfasis en la naturaleza y alcance de la mediación cognoscitiva que se realiza en contextos de educación no formal, como las bibliotecas, archivos y centros de información y documentación, entre sujetos emisores-productores de información y conocimiento y sujetos receptores y activos constructores de significados a partir de esa mediación. Igualmente, la metodología considera el análisis de contenido para desvelar las concepciones que sobre el proceso manejan expertos de la UNESCO y la IFLA, con el propósito de ayudar a elaborar renovadas aproximaciones teóricas sobre la función de las organizaciones 
del conocimiento, frente al desafío impuesto por los cambios sociales, científicos y tecnológicos.

\section{DESARROLLO TEÓRICO}

\section{Construcción conceptual y caracterización}

de la comunicación-mediación en organizaciones de conocimiento

Este estudio parte de la necesidad de generar explicaciones teóricas que sirvan de referente para la acción de las organizaciones de conocimiento frente a los desafíos actuales. Se asume la premisa de que las categorías conceptuales propias de las ciencias de la información y la comunicación se muestran inadecuadas para explicar la naturaleza y el alcance de los fenómenos de la información y el conocimiento a la luz del surgimiento de un nuevo espacio-tiempo, que otorga contexto a una estructura socio-cultural signada cada vez más por el uso extensivo de las tecnologías telemáticas e interactivas, lo cual obliga a repensar los conceptos de sociedad y de hombre que se han utilizado históricamente en las ciencias de la información y la comunicación, vinculadas a su vez con las ciencias sociales y humanas.

La sociedad se representa en este trabajo a partir de las metáforas del tejido, la red y lo entrecruzado, porque su funcionamiento se organiza en función de conformar no sólo redes electrónicas, sino sociales y humanas, es decir, grupos de individuos que produzcan conocimiento de manera cooperadora y compartida. El sujeto se concibe en un tránsito del homo sapiens, al homo digitalis (Terceiro, 1996; Beckett, 2000) porque se convierte en un bios virtual (Sodré, 2001), por lo que se requiere emprender revisiones conceptuales e integraciones de enfoques para aproximarse a la generación de nuevas líneas teóricas mucho más acordes con las actuales condiciones de la sociedad y el hombre digital.

Se considera que frente al planteamiento de una sociedad de las redes, la comunicación y el conocimiento, es necesario proponer otros sistemas de conceptos que guíen las prácticas en las organizaciones de conocimiento, porque estas organizaciones deben desempeñar un rol protagónico en el aprendizaje permanente de los ciudadanos, mediante procesos de mediación cognoscitiva; aunque históricamente a estas or- 
ganizaciones se les han planteado problemas que van de lo conceptual a lo infraestructural e instrumental, lo que ha incidido negativamente en el logro de sus funciones medulares.

Los problemas conceptuales que han impedido la realización efectiva del proceso de comunicación-mediación, por parte de las organizaciones de conocimiento, están asociados con la concepción y percepción social que se tiene de éstas y de los profesionales que las dirigen. Por ello, para reposicionar la acción que desde estos espacios se debe realizar, es necesario adoptar drásticas decisiones sobre la concepción misma de las profesiones y los profesionales que las representan. Esta concepción, según nuestro criterio, debe verse más allá de una relación fría entre los sujetos emisores y los receptores, mediante el préstamo, suministro de información, o realización de actividades y procesos técnicos, rutinarios y tradicionales, para dar cabida a una dimensión más dinámica y compleja, vinculada con la comunicabilidad, la relación, la mediación y facilitación no de documentos, sino de conocimientos, lo cual permitirá optimar los procesos de comunicación de la inteligencia social en el contexto regional, nacional e internacional (Páez, 1992:36).

Los problemas conceptuales han originado limitaciones que tienen que ver con la manera como se ha entendido la acción de estas organizaciones, lo cual ha influido en el grado de desarrollo de los servicios de información que se prestan y, por ende, en la satisfacción de las necesidades y requerimientos informativos y cognoscitivos de los usuarios receptores.

Desde el punto de vista infraestructural, las organizaciones de conocimiento a las cuales hacemos referencia presentan actualmente problemas que van desde no contar con infraestructuras físicas planificadas sobre la base de criterios científico-técnicos, hasta dificultades derivadas de la baja asignación presupuestaria, y la formación y actualización de personal. En Venezuela, tales situaciones se repiten, lo cual puede verse en diversos diagnósticos regionales y nacionales sobre la situación general del sector información (bibliotecas y archivos). Particularmente en el Estado Zulia, los últimos informes presentados en este sentido son los de Primera (1992) y Morillo (2000), que se han referido de modo particular a los signos de crisis de la red de bibliotecas públicas del estado.

En cuanto a los archivos, la situación no sólo se repite, sino que tiende a agudizarse, ya que es evidente el rezago que muestran estas 
instituciones en su ingreso a los nuevos entornos tecnológicos y el diseño de servicios de información con valor agregado. Se ha llegado a la conclusión que dentro del sector información, las organizaciones más desasistidas en Venezuela son los archivos (Niño, 1994, Ortega y otros, 1999).

Si volvemos la mirada a ámbitos internacionales no encontramos cifras que representen cómo se está dando el proceso de comunicaciónmediación en organizaciones de conocimiento en concreto, pero sí existen resultados que apuntan hacia las tendencias generales que en las diversas regiones del mundo se manejan en torno a estas organizaciones y las nuevas funciones que deben cumplir.

En el caso, por ejemplo, de la industria de la información (sector bibliotecas) en Europa Occidental, se apunta en este momento hacia una revisión de los modelos de bibliotecas basadas en la categoría de usuarios. En otras palabras, la distinción entre biblioteca universitaria y pública empieza a desaparecer, debido a que hoy es posible difundir la información desde y a través de todos los puntos de la red. Esto necesariamente implica que estas organizaciones realicen diferentes funciones en el nuevo entorno electrónico, encaminándose hacia nuevas formas de producción y de distribución de la información (Vitiello, 1997).

En el caso de América del Norte, se observa que los servicios de información están bien desarrollados y se enfrentan a cambios que resultan de la revolución tecnológica. El sector información de esta región se preocupa en la actualidad por aprovechar la oportunidad que ofrece la aparición de la biblioteca digital, al tiempo que existe una evidente preocupación por la cooperación entre diferentes organizaciones de información (bibliotecas y archivos). El personal desempeña tareas de especialistas de información, ayudando a los usuarios a encontrar informaciones cruciales y enseñándoles a localizarlas por ellos mismos. (Moore, Hajnal y Manning, 1997).

En las dos últimas décadas, América Latina y el Caribe, en general, han experimentado un desarrollo favorable relacionado con la calidad y variedad de sus servicios de información, así como con la infraestructura tecnológica, colecciones y recursos humanos profesionales. Este desarrollo va en paralelo con el avance socio-económico y cultural de cada país (Morales, 1997:128). 
Pero la realidad de las organizaciones de información de los países desarrollados y los denominados del Tercer Mundo muestra una brecha que distancia los planteamientos de la cibersociedad de nuestros países y realidades.

Autores como Trejo (1999) y Agudo (2001) plantean que nuestras sociedades no son sociedades del conocimiento, porque no se observan los rasgos distintivos de las sociedades reconocidas como tales. Algunos de esos rasgos son: a) La sustitución de materias primas, insumos y bienes industriales por la producción y comercialización de información y conocimiento. b) El nacimiento y crecimiento de una industria de información, entendiendo por la misma la conformada por la producción de contenidos informativos (datos, noticias, información y conocimiento), los canales para que circulen y las tecnologías que permitan su integración en procesos comunicativos. c) La incorporación de las tecnologías de información y comunicación (TICs) por los actores sociales en procesos de gerencia social, económica, política y comunitaria. d) El acceso de todos los niveles y sectores sociales a las TICs. e) La participación del Estado, la sociedad civil y la empresa privada en la construcción de la infraestructura de información; y f) que la mayoría de la población haya sido alfabetizada en teleinformática.

Según ambos autores, además de no poseer la mayoría de los anteriores rasgos, en nuestro contexto, la llamada globalización introduce y agudiza los desequilibrios sociales culturales.

Sin embargo, habría que matizar que aunque no es posible hablar de que las sociedades latinoamericanas en su totalidad están participando plenamente de los beneficios de la globalización, sí es posible señalar que algunas de ellas se pueden ir incorporando a políticas y programas que permiten avanzar hacia la sociedad del conocimiento y la comunicación, lo cual dependerá del grado de desarrollo bajo, medio y emergente que manifiesten.

La presencia escasa, media o total de los rasgos distintivos de la sociedad del conocimiento, impacta positiva o negativamente el proceso de comunicación-mediación del conocimiento, sobre todo en este momento histórico, en el cual no es posible hablar de comunicación y de conocimiento sin considerar la inclusión de las tecnologías telemáticas, 
lo cual pasa por enseñar a utilizar los recursos informativos y cognoscitivos y los nuevos medios existentes para expresarlo.

El fenómeno de la globalización tiene, como todos los fenómenos y tendencias, detractores y seguidores. Algunos de los argumentos que esgrimen detractores como Vilas (2001) tienen que ver con la refutación de las cinco ideas centrales que sustentan esta ideología, a saber:

1. Que la globalización es un fenómeno nuevo.

2. Que es, asimismo, un proceso homogeneizador: ya que todos seremos, antes o después iguales y, en particular, los latinoamericanos seremos iguales en desarrollo, cultura y bienestar a nuestros vecinos del norte y de Europa.

3. Que la globalización conduce al progreso y al bienestar universal.

4. Que la globalización de la economía conduce a la globalización de la democracia.

5. Que la globalización acarrea la desaparición progresiva del Estado, o al menos una pérdida de importancia del mismo.

El investigador argentino refuta cada una de estas ideas, recurriendo a cifras y evidencias que constatan el comportamiento de la globalización en América Latina y señala que la globalización es un conjunto de procesos, que viene desarrollándose con aceleraciones y desaceleraciones a lo largo de los últimos cinco siglos. Estos procesos tienen dinámicas y ritmos desiguales y su efecto coordinado es profundamente diferenciador, tanto dentro de los espacios económicos nacionales y regionales como entre las regiones del mundo. Dadas ciertas condiciones ligadas a un conjunto amplio de factores socioeconómicos, culturales y políticos, la globalización puede redundar en oportunidades de mayor bienestar social, progreso técnico y desarrollo económico, pero en otras condiciones puede generar efectos exactamente opuestos.

Junto a estas visiones del fenómeno de la globalización conviven otras que plantean matices, como las de Ortiz (2002), quien diferencia entre dos conceptos para abordar las características de una sociedad global, a saber: el de globalización, que se relaciona con fenómenos económicos-tecnológicos, y el de mundialización, que alude a lo cultural. A partir de ellos, el autor afirma que existe un proceso de globalización, pero a 
nivel económico y tecnológico; sin embargo, en el plano cultural no hay una globalidad, sino una mundialización de la cultura, que tiene especificidades distintas de la globalización económica y tecnológica.

En relación con lo social, el autor señala que a partir de la globalización se introducen modos de exclusión pero también de inclusión, de manera que es importante investigar cómo las clases subalternas son excluidas o incluidas de forma desigual en este proceso, es decir, sería interesante advertir las reacciones de estas clases dentro de la esfera del consumo, que están presentes en su imaginario (televisión, sueños), pero al mismo tiempo les son negadas en la práctica (por falta de ingresos para poder participar).

Ortiz (2002) también plantea que pensar la globalización de las sociedades no es otra cosa que la afirmación de la existencia de procesos que comprenden a los grupos, las clases sociales, las naciones y los individuos, los cuales interactúan en un contexto mundializado en el que operan cambios culturales amplios, es decir, transformaciones en la producción, el tiempo, la memoria, la noción de territorio y las interrelaciones entre las personas. Estos cambios y transformaciones se están dando como consecuencia de las tecnologías de información y comunicación, ante lo cual se les impone a las instituciones sociales como la escuela, y a otras como las organizaciones de conocimiento, asumir roles mediadores, como orientar, dialogar y guiar a los ciudadanos.

De manera que nos encontramos frente a los desafíos de una sociedad que tiende a fundarse más en el conocimiento. Teóricos, como Drucker (1993), plantearon un nuevo tipo de sociedad, la llamada sociedad del conocimiento, reconocida como el paso de la sociedad capitalista a una pos-capitalista, en la que los servicios y el conocimiento se convierten en recursos estratégicos para impulsar el desarrollo individual y social.

Esta sociedad también se conoce como sociedad de la comunicación, pues si en los noventa se habló de una sociedad de información, al inicio de un nuevo siglo se está reconociendo que la información no es lo más importante, sino lo que hacen con ella los actores en interacción dialógica (Galindo, 1998); igualmente se está hablando de que la comunicación es el vector que permite que la información-saber fluya y facilite la expansión y dinamice la inteligencia humana (Pineda y otros, 2001). 
De manera que a pesar de las asimetrías que se observan en la región latinoamericana, en relación con los procesos de acceso al conocimiento soportado en tecnologías de información y comunicación, son innegables, no se pueden desdeñar las tendencias señalas por los teóricos de las ciencias de la comunicación, que plantean el surgimiento de una sociedad que, en futuro inmediato, se valga de la información-saber disponible en sistemas, bases de datos y en la red, para expandir la inteligencia humana y las oportunidades de intervención ventajosa de los diferentes contextos donde los sujetos se insertan.

\section{Hacia una tipología de las mediaciones}

en las organizaciones de conocimiento

El estudio que emprendemos considera la comunicación del conocimiento como un proceso complejo de intercambios recíprocos de mensajes informativos y conocimientos, los cuales son posibles a partir de la realización de tres tipos de mediaciones: la primera es la que hace el sujeto investigador-productor del conocimiento, que se basa en su arsenal cognoscitivo y de representación, y se apoya en medios electrónicos de comunicación, mediante los cuales se utilizan modelos de escritura digital e interactiva, que hacen surgir nuevas lógicas mucho más holísticas e integradoras en el proceso de producción de las informaciones y los conocimientos. La segunda mediación es la que realiza el profesional de la información en el contexto de la organización de conocimiento, quien aplica a la información generada procesos de organización y representación, los cuales deben estar centrados más que en el análisis documental formal, esto es en procesos informativos-descriptivos, en procedimientos de análisis documental de contenido, lo cual implica incorporar la perspectiva cognoscitiva y semántica a los contenidos que expresan el conocimiento. Estos procedimientos buscan producir información con valor agregado y constituyen la base de la construcción de sistemas digitales de comunicación del conocimiento. Éstos son procesos previos a la mediación posterior que hace el sujeto receptor activo, quien realiza la tercera mediación, a partir de la cual expande su estructura y arquitectura cognoscitiva, al incorporar nuevos conocimientos, logrando con ello, lo que plantea Popper (1992) en relación con el conoci- 
miento "acerca del mundo", es decir, con la mediación que el sujeto receptor activo realiza partiendo de las otras dos mediaciones, es posible expandir los conocimientos que los sujetos necesitan para utilizarlos en la acción, específicamente en la resolución de problemas, la toma de decisiones y el emprender retos.

A partir de la explicación de las tres mediaciones es posible hablar entonces de comunicación-mediación del conocimiento y no de transferencia de información, de prestación de servicios de información, gestión de información o de gerencia del conocimiento, categorías conceptuales utilizadas con frecuencia en la literatura especializada en bibliotecología, archivología y documentación para referirse al proceso medular que deben realizar las organizaciones de conocimiento.

Proponemos el concepto de organizaciones de conocimiento, considerando como criterio de categorización, el tipo de información con la cual se trabaja en estas organizaciones, ya que se trata de informaciónsaber (Pineda, 2000), es decir, la información en tanto que objetivación del pensamiento y el intelecto humano. Igualmente nos fundamentamos en la visión de Popper (1992), quien asume el conocimiento según dos categorías generales: el subjetivo, que supone la presencia de un sujeto cognoscente, y el objetivo, materializado y articulado en teorías. Paralelo a ello, estos dos conocimientos interactúan en tres mundos: el mundo 1 , correspondiente a lo físico; el mundo 2, relacionado con las experiencias conscientes, y el mundo 3, el de los libros, bibliotecas y bases de datos e informaciones. La conclusión de la teoría popperiana es que "nuestro conocimiento del mundo" depende del mundo 3, a lo que se le puede añadir, de las acciones comunicativas-cognoscitivas desarrolladas desde el mundo tres.

\section{Revisión de enfoques conceptuales sobre}

la comunicación-mediación en organizaciones conocimiento

La tesis que presentamos, relacionada con la función de las organizaciones de conocimiento en la cibersociedad, se enmarca en una perspectiva comunicativa, lo cual no es novedoso en cuanto a que ya otros autores de los ámbitos bibliotecológicos, archivológicos y documentalistas se han referido a ésta y a la necesidad de entender la función de las institu- 
ciones y organizaciones de conocimiento como una acción comunicativa; de hecho, el propósito central de éstas organizaciones es precisamente lograr la comunicación de los conocimientos. Lo novedoso de nuestros planteamientos está en la integración de perspectivas comunicacionales, informacionales y cognoscitivas, para generar una nueva línea teórica explicativa sobre la acción de estas organizaciones.

Dentro de los autores que se refirieron al "hacer" de las organizaciones de conocimiento, desde una perspectiva comunicativa, tenemos a García (1992), quien abordó los aspectos teóricos y organizacionales del proceso de comunicación en las unidades documentales, a partir de una investigación que estableció relaciones entre la información, la comunicación y la documentación, considerando el análisis de sus objetivos, funciones y elementos que las conforman, y analizando también los modelos y teorías de la comunicación de Laswell, Katz, Lazarsfeld, Riley, Mc. Luhan, Moles, Gerbner y Balle, con el fin de explicar el proceso de comunicación en las unidades documentales. El estudio concluyó en la necesidad de organizar los servicios informativos y documentales en función de los tres niveles de apertura comunicacional (difusión, divulgación y diseminación).

Si revisamos una de las primeras propuestas conceptuales de la perspectiva bibliotecológica, encontramos las ideas de Shera (1972), quien, al asumir una concepción comunicativa y mediadora de la acción del bibliotecólogo, reconoció que la biblioteca se inserta en el sistema general de comunicación, que debe actuar en dos macrocosmos: el de la cultura, en donde reside la producción del conocimiento, y el del individuo, caracterizado a partir de las necesidades e intereses puntuales de información.

En la perspectiva documentalista de Otlet (1934) también es posible ubicar planteamientos tempranos de una visión comunicativa y cognoscitiva, ya que, según el autor, el propósito del documentalista está en acortar la distancia entre el conocimiento producido y los usuarios, mediante el despliegue de refinados procedimientos informativos-documentales, centrados en la extracción y potenciación de los contenidos semánticos presentes en los documentos. Aunque este enfoque se afinca en la importancia de los soportes documentales, plantea un concepto universal e integrador de documento y hace énfasis en 
el carácter dinamizador de la documentación, entendida como una disciplina que busca extraer la información contenida en los diversos soportes de los documentos para maximizar su uso, es decir, garantizar su comunicabilidad para que, posteriormente, pueda utilizarse la información en la acción.

La perspectiva informacionalista es otra de las que fundamenta este estudio, principalmente la defendida por el Georgia Institute of Technology (Cronin, 1983; Páez,1992). Esta perspectiva asume que la información es un elemento complejo, que contiene propiedades, medios y procedimientos utilizados para su transformación y movimiento; plantea el carácter estratégico y decisivo del uso adecuado y efectivo de los recursos informativos en las organizaciones, por lo que es una perspectiva que se mueve más en el plano de la información, en tanto flujo de datos, que de soportes documentales. En el estudio se incluyen también conceptos medulares de la tendencia emergente, referida a la gerencia del conocimiento, la cual, a la luz de esta investigación, se ubica en los enfoques teóricos de las ciencias de la información, particularmente aquéllos relacionados con el aspecto gerencial de la información, que plantean el paso de una gestión de la información a una del conocimiento. Tales conceptos medulares son los de conocimiento explícito y conocimiento tácito, señalados por Nonaka y Takeuchi (1998).

Como se trata de construir una urdimbre teórica que aporte elementos para la interpretación del proceso de comunicación-mediación en organizaciones de conocimiento, no sólo se consideran en el estudio algunos fundamentos conceptuales de las ciencias de la información, sino de la comunicación y las ciencias cognoscitivas. En cuanto a las ciencias de la comunicación, se asume un concepto de mediación que hunde sus raíces en las ideas de Martín Serrano (1997), Martín-Barbero (1998) y MuñizSodré (2001), a partir de las cuales es posible entender la comunicación en tanto posibilidad de mediar entre sujetos emisores y sujetos receptores; en el caso de la cibersociedad, ese espacio de la mediación es telemático, por lo tanto surgen tecno-interacciones y se fomentan renovadas pautas de recepción activa, procesamiento de información y conocimiento para tomar decisiones, resolver problemas y emprender retos.

Por otro lado, la investigación también incorpora los nuevos conceptos y categorías que surgen en las formas emergentes de comunica- 
ción, señaladas por Cartier (1992), Cloutier (1992), Pineda (2000) y Castells (1999), y las cuales se desplazan de la verticalidad defendida desde los modelos funcionalistas de la comunicación a la horizontalidad que supone los conceptos de comunicación interactiva, dialógica, telemática y audioescriptovisual, que rompe con la visión tradicional de tiempo y espacio y convierte al proceso comunicativo, mediado por las nuevas tecnologías de comunicación e información, en un fenómeno complejo, estético y globalizador, el cual requiere de nuevas habilidades, destrezas y competencias por parte de los actores participantes en el proceso.

En cuanto a los elementos teóricos de las denominadas ciencias cognoscitivas, la perspectiva teórica integrada que proponemos se complementa con los conceptos de arquitectura y fluidez cognoscitiva de Mithen (1998), quien piensa que la mente humana se construye mediante un proceso muy parecido al de las catedrales y que depende tanto del código impreso en el mapa genético de cada persona, pero también de las interacciones que haga en ámbitos formales y no formales de aprendizaje; la conformación de las arquitecturas mentales surge como producto de la fluidez cognoscitiva, aspecto que garantizará a las personas el adecuado y efectivo desenvolvimiento en diferentes contextos.

\section{ANÁLISIS DOCUMENTAL: RESULTADOS PRELIMINARES}

Visión de UNESCO e IFLA en torno a la comunicación-mediación en organizaciones de conocimiento: algunas tendencias

Para detectar las tendencias conceptuales que se manejan en los ámbitos internacionales sobre el proceso de comunicación-mediación en las organizaciones del conocimiento de la cibersociedad, se analizaron los contenidos de ocho documentos internacionales, escogidos según criterios consistentes con el enfoque teórico-metodológico que enmarca el estudio. Los documentos son: La Declaración de Copenhague (1999), la Declaración de Educadores e Investigadores de Bibliotecología y Ciencia de la Información de Iberoamérica y El Caribe (2003), la Declaración de Itacurucá (2000), la Declaración de Florianópolis (2000), el Acta del Primer Taller Latinoamericano y del Caribe de Tecnologías de Infor- 
mación y Comunicación (2000), el Manifiesto de la UNESCO sobre la Biblioteca Pública (1994) y el de la Biblioteca Escolar (2000) y el Acta de Relatoría del XIV Congreso Internacional de Archivos realizado en Sevilla, España (2000).

A estos documentos se les aplicaron dos tipos de análisis. Un análisis consistió en cotejar la presencia y la ausencia de las relaciones teórico-conceptuales construidas para el estudio, con el propósito de detectar hasta qué punto en estos documentos, en tanto directrices para la acción, se están manejando concepciones integradoras y holísticas que vinculan la acción de las organizaciones de conocimiento a partir de la comunicabilidad, el aprendizaje y la cognición. El otro tipo de análisis consistió en desvelar las concepciones que sobre el hombre, la sociedad, la acción de los profesionales y los servicios y productos se manejan en los documentos. Ambos análisis permitieron saber el grado de consistencia, congruencia que existe por parte de la comunidad de especialistas en ciencias de la información en torno a los conceptos medulares que deben guiar las "nuevas prácticas" de las organizaciones de conocimiento, a modo de poder ajustarse a los cambios presentes y futuros

Tales análisis se realizaron mediante la construcción de matrices de cotejo y desmontaje de contenidos, en las cuales se consideraron categorías como: concepción de la sociedad, del hombre, del conocimiento; concepción de las organizaciones de conocimiento, concepción de los usuarios, competencias propuestas para el profesional de la información, procesos y productos a los que se hace referencia para lograr la comunicación del conocimiento.

Luego de cotejar y analizar los documentos se concluye que, aunque UNESCO, IFLA y los especialistas en archivología de diferentes países no hacen alusión a la diferencia conceptual importante que existe entre la sociedad de la información y la del conocimiento, más aún, se refieren a la información y al conocimiento como categorías conceptuales análogas, sin embargo, han elaborado una red de relaciones teóricas que hacen énfasis en la emergencia de un nuevo tipo de sociedad que demanda, de parte de las organizaciones de conocimiento y sus profesionales, la realización de esfuerzos de actualización y formación permanente en materia de tecnologías de información y comunicación, como apoyo esencial de la infraestructura de servicios, procesos y actividades. 
En cuanto a la concepción del hombre, se reitera en varios documentos el carácter humanista, progresista y democrático del individuo para garantizarle su incorporación activa en la sociedad del conocimiento. Se insiste en plantear la reducción de las brechas individuales y sociales, sobre la base de una concepción igualitaria de los sujetos. Si se logra una igualdad en el acceso, se construiría una sociedad igualitaria, entendiendo que la vía para lograrlo es a través de la equidad en el acceso a los recursos informativos, para lo cual las organizaciones de conocimiento deben asumir un rol protagónico.

En relación con la idea de sociedad que subyace en los documentos, es evidente el carácter múltiple, complejo y cambiante que se le quiere atribuir a la sociedad, entendida como conglomerado articulado a partir del uso masivo de los medios telemáticos e interactivos. En este sentido, observamos congruencia entre los planteamientos expuestos por teóricos e investigadores de la comunicación y la información, y las ideas de los especialistas de UNESCO e IFLA. Sin embargo, llama la atención la falta de acuerdo en cuanto a la denominación conceptual de la sociedad actual, al punto de pretender homologar el término sociedad de la información con el de sociedad del conocimiento, lo cual es una inconsistencia que requiere estudio y profundización, ya que no es igual plantear una sociedad de la información a una del conocimiento. Siguiendo a Pasquali (2003), la información no crea sociedad, sólo la comunicación-diálogo y la construcción colectiva del conocimiento hace posible eso; además, tener información no equivale a un desarrollo de la racionalidad y el conocimiento, es sólo una parte, ya que el conocimiento implica reflexión acerca de los datos e informaciones y requiere de la aplicación de procesos innovadores de agregación de valor. Es por ello que, en la investigación, se diferencian las concepciones de sociedad de la información y del conocimiento, tomando en cuenta las diferencias entre los conceptos información, conocimiento y comunicación y lo que implican para las organizaciones de conocimiento, sus funciones y actores.

En los documentos revisados no existe una concepción clara de lo que es el conocimiento. Sin embargo, se da por sentado que es un recurso medular para impulsar el desarrollo educativo, científico, tecnológico y cultural. También se expresa que el conocimiento y la "información", sus usos adecuados, incrementan la calidad de vida de los ciudadanos. Las 
expresiones "nueva era del conocimiento", "economía del conocimiento", hacen pensar que indudablemente los especialistas que elaboraron los documentos están conscientes de que nos encontramos en un momento histórico caracterizado cada vez más por el uso estratégico que se haga del conocimiento; incluso se llega a plantear que es el punto de partida para la construcción de la democracia y la participación.

En cuanto a la concepción de las organizaciones de conocimiento, se les denomina de diferente manera: instituciones culturales, instituciones de memoria, centros de información, promotoras del aprendizaje, de lo cual se deduce la incompatibilidad de criterios teóricos para definir conceptualmente a tales organizaciones. El tener claridad sobre la denominación que se debe dar a estas organizaciones indudablemente orientaría las nuevas funciones que se deben cumplir. Aunque la unificación conceptual es una tarea pendiente por parte de los especialistas, existe consenso en el hecho de que son otras las prácticas, los procesos y actividades que se deben realizar. La idea de sinergia, cooperación, colaboración y estructuración en redes e infraestructuras de información, propone formas de trabajo intra e interorganizacional que se corresponden con la lógica reticular presente en la sociedad actual. Es importante mencionar también que aunque se manejan nuevas epistemes sobre las organizaciones, permanecen nociones asociadas a la conservación y resguardo, lo cual plantea la presencia de una visión física de estas organizaciones, en el sentido de los soportes y espacios utilizados, lo cual constituye igualmente una inconsistencia si se asume que en la cibersociedad asistimos a un desplazamiento de la idea de biblioteca o centro de información, concebido mediante la lógica de los documentos físicos e impresos, hacia la conformación de organizaciones de conocimiento con colecciones digitales, en el contexto del ciberespacio.

En los materiales que fueron analizados existe acuerdo en cuanto al uso de los recursos hipermedia, virtuales e Internet para impulsar el desarrollo económico y social, así como también para acometer procesos de alfabetización digital y teleinformática. Igualmente, existe consenso en cuanto a la concepción del usuario, el cual debe entenderse como un sujeto que requiere la información y el conocimiento para su desarrollo como persona, como ciudadano y agente activo que debe participar en el contexto de la globalización. 
Asimismo, existe acuerdo en el alcance de las organizaciones de conocimiento, en relación con la posibilidad de ofrecer alternativas para el aprendizaje tecnológico-informativo y el deutero-aprendizaje, concebido este último como el aprender a aprender, es decir, desarrollar las competencias para "saber conocer". Esta afirmación se apoya en la idea impulsada también por UNESCO, referida a la educación a lo largo de toda la vida, lo cual constituye el eje de las implicaciones educativas que tiene el proceso de comunicación-mediación en las organizaciones de conocimiento hacia el futuro.

En cuanto a las competencias del profesional de la información, se insiste en plantear la redefinición de funciones, servicios y procesos, los cuales deben ser de "alto" valor agregado; de esto se desprende el necesario desarrollo de nuevas funciones y procesos, relacionados con la gestión de información, la educación de los usuarios para la interacción tecnológica y el uso efectivo de los recursos de información, y la participación activa en el proceso de producción de conocimiento. Lo último constituye una tendencia marcada con mucha fuerza; el profesional no es sólo mediador, sino productor y generador de conocimientos, soportados en tecnologías, lo cual otorga matices diferentes al trabajo del profesional de la información, ya que incluso a la luz de los cambios técnicos y sociales, se había propuesto que el profesional debía desarrollar funciones de gestor, consultor y asesor, pero no de creador de conocimientos.

Finalmente, en relación con los procesos, productos y servicios a los que se hace referencia para realizar la mediación del conocimiento, se tiene que en los documentos, en muchos casos, no se especifica cuáles y qué naturaleza deben tener tales procesos, productos y servicios. Sin embargo, se detectó que en otros casos sí se llega a explicitar cuáles son los procesos, productos y servicios. En relación con los procesos, se habla de gestión de información, organización de información y conservación, realización de investigaciones sobre las tecnologías de información y comunicación, conformación de redes de organizaciones, infraestructuras nacionales que faciliten el acceso al conocimiento, organizadas a partir de políticas y legislaciones específicas. En cuanto a los productos y los servicios, existe consenso en plantear la producción de contenidos locales, regionales y nacionales generados en lenguas nativas y oficiales de la región, los cuales deberán alojarse en servidores regionales, es decir, se 
habla de diseño de portales y sistemas de conocimiento sobre contenidos de uso público, referidos a las regiones, como una forma de participar en la dinámica del conocimiento en la cibersociedad.

En definitiva, las tendencias conceptuales y de acción propuestas desde los documentos internacionales son las siguientes:

1. En la sociedad del conocimiento, los ciudadanos deben tener acceso equitativo a la información que se produce, como una forma de reducir las posibles brechas sociales que pueden generarse en el contexto de la globalización.

2. Se reconoce el carácter sistémico, reticular e integrado de la sociedad.

3. Las organizaciones de conocimiento (bibliotecas, archivos, centros de documentación) tienen un rol protagónico en la formación de los ciudadanos para la inserción en la sociedad del conocimiento, lo cual implica enseñar a utilizar los recursos telemáticos e interactivos. En este sentido, se ratifica una concepción de facilitación informativa y educativa de las organizaciones. Comienzan a introducirse ideas sustentadas en la comunicabilidad y lo cognoscitivo.

4. Las diversas y complejas acciones humanas deben apoyarse cada vez más en el conocimiento y en las tecnologías de información y comunicación, reconociendo con ello la utilidad de las tecnologías para el desarrollo y el equilibrio social.

5. Las concepciones, funciones y procesos que realizan las organizaciones de conocimiento deben centrase en el usuario, quien necesita saber utilizar la información, el conocimiento y las tecnologías para impulsar su desarrollo personal, social y profesional.

6. De lo anterior se desprende que las organizaciones de conocimiento tienen el compromiso de desarrollar el autoaprendizaje para toda la vida y el aprendizaje tecnológico informativo.

7. Las competencias del profesional de la información deben orientarse hacia el tratamiento creativo e innovador de la información, para generar "alto valor" agregado, así como también se requieren nuevas competencias para la creación y difusión del conocimiento mediante las tecnologías telemáticas e interactivas.

8. Partiendo de la tendencia anterior, los procesos, productos y servicios que se necesitan para comunicar-mediar entre el conocimiento 
y los usuarios son: los estudios de necesidades, el diseño de contenidos para el cibersespacio y la educación y alfabetización teleinformática.

\section{PROPUESTA TEÓRICO-METODOLÓGICA DEL ESTUDIO}

\section{Aproximación a una línea teórica integrada sobre}

la comunicación-mediación en organizaciones de conocimiento:

el contexto de su construcción

Luego de cotejar la construcción y caracterización del proceso de comunicación-mediación en organizaciones de conocimiento, de revisar los enfoques teóricos que lo fundamentan y analizar los documentos internacionales en donde se expresan sus tendencias, es posible concluir que el planteamiento central del cual se desprende esta integración de perspectivas es que el carácter dinámico y estratégico de la acción comunicativa-cognoscitiva, en tanto acción mediada que se debe realizar en el contexto de las organizaciones de conocimiento, podría propiciar el aprendizaje tecnológico-informativo y permanente de las personas. Pudiendo transformar así sus estructuras de pensamiento y potenciar su fluidez cognoscitiva, de manera que puedan utilizar el conocimiento aprendido en la acción y avanzar hacia procesos educativos más abiertos y democráticos, lo cual supone participación activa y desarrollo.

Estos planteamientos permiten proponer algunos supuestos para justificar la construcción de una línea teórica integrada y articulada de las ciencias de la información, la comunicación y las ciencias cognoscitivas; tales supuestos son:

1. El paso de la sociedad de la información a la cibersociedad, reconocida como una sociedad del conocimiento y la comunicación obliga a redimensionar la acción de las organizaciones de información de lo instrumental-técnico y lo facilitador-informativo a lo comunicativo-cognoscitivo.

2. Es necesario interpretar la acción de esas organizaciones desde la comunicabilidad, entendida como posibilidad de mediación del conocimiento. 
3. El proceso que articula la acción es el de comunicación-mediación del conocimiento, expresión conceptual que implica mucho más que transferencia de información, comunicación documental y gestión de información.

4. La comunicación del conocimiento es un proceso que se vuelve complejo, ya que se introducen formas interactivas y dialógicas que rompen con las nociones tradicionales de tiempo, espacio, documento e información; tales formas, si se saben aprovechar, impulsan el aprendizaje y el desarrollo.

El proceso de construcción de la línea teórica integrada se sustenta en el enfoque de Bagozzi y Phillips (1982), conocido como constructo holístico, mediante el cual es posible representar y probar teorías organizacionales. El mismo se asume como una metodología para examinar la validez de los conceptos y sus mediciones, y también para predecir y probar hipótesis. El enfoque, que parte de la premisa de que una investigación tiene mayor validez si se encuentra guiada por un pensamiento conceptual bien fundado, tiene sus orígenes en la conceptualización de sistemas teóricos de Hempel (1952), quien plantea que la teoría científica puede ser comparada con una red compleja, sus términos están representados por los nudos, mientras que las cuerdas que conectan los últimos corresponden en parte a las definiciones y en parte a los fundamentos e hipótesis derivadas e incluidas en la teoría. El sistema completo flota sobre el plano de observación.

El constructo holístico se apoya también en la idea de teorías científicas de Popper (1973), según la cual, tales teorías son redes que se lanzan para apresar el mundo con el objeto de racionalizarlo, explicarlo y dominarlo. Se trata que la malla sea cada vez más fina. La razón científica, según esta perspectiva, funciona mediante la construcción de sistemas peculiares de conocimiento del mundo, con el objeto de poder explicar los fenómenos y no sólo describirlos.

El enfoque de Bagozzi y Phillips (1982) indica tres tipos de conceptos y cuatro relaciones. En cuanto a los conceptos se identifican los conceptos teóricos, los derivados y los empíricos; y las relaciones son: las hipótesis no observables, la definición teórica, la regla de correspondencia y la definición empírica. 
Los conceptos teóricos son abstractos y no observables, consisten en descripciones de fenómenos proporcionados por frases que reflejan el vocabulario conceptual de la teoría. Los conceptos derivados, al igual que los teóricos, son no observables, pero están atados directamente a los conceptos empíricos y se encuentran en un nivel de abstracción menor que los conceptos teóricos. Los conceptos empíricos se refieren a las propiedades o relaciones, cuya presencia o ausencia, en una situación dada, puede ser conocida intersubjetivamente bajo circunstancias apropiadas, mediante la observación directa.

En cuanto a las relaciones del enfoque holístico, se encuentran las hipótesis no observables que unen dos o más conceptos teóricos, mediante un enunciado. En el caso de nuestro estudio, una hipótesis no observable puede ser: en la cibersociedad, las organizaciones de conocimiento deben articular su acción mediante un proceso de comunicación-mediación, a partir del cual se potencia el aprendizaje en los usuarios receptores activos. Puede observarse que los tres conceptos teóricos que se conectan son: cibersociedad, comunicación del conocimiento y aprendizaje.

La segunda relación que se propone es la definición teórica, que conecta un concepto teórico con uno derivado, mediante una definición nominal. La tercera relación es la regla de correspondencia que expresa una relación entre conceptos no observables (teóricos y derivados) y conceptos empíricos, y la cuarta relación es la definición empírica que se caracteriza por dar significado a un concepto empírico igualándolo con eventos físicos actuales en el mundo de la experiencia de los sentidos.

La línea teórica integrada se propone a partir de la intersección de las tres ciencias implicadas en la explicación de los procesos de comunicación-mediación del conocimiento, lo cual se muestra en el modelo de intersección disciplinar (reticularidad teórica) que se anexa, del cual emergen nuevos conceptos que se representan mediante una malla teórica y se recogen en un cuadro explicativo.

\section{CONSIDERACIONES FINALES}

Luego de aproximarnos a los elementos teórico-epistemológicos que se están trabajando para la investigación, es posible puntualizar en las siguientes consideraciones: 
1. Los cambios y transformaciones producidos por las tecnologías de información y comunicación están obligando a replantear no sólo las formas de organización y mediación social y cognoscitiva, sino también los conceptos utilizados en las ciencias que dan soporte teórico-conceptual explicativo a los sistemas y procesos de comunicación-mediación. De allí la necesidad de asumir enfoques integrados para explicar la comunicación-mediación del conocimiento en la cibersociedad.

2. La investigación en ciencias de la información y comunicación tiene el reto de producir transformaciones que incluso lleguen a repensar las estructuras epistemológicas predominantes en la producción del conocimiento científico, lo cual supone la utilización de estrategias metodológicas consistentes con los fenómenos que se estudian, caracterizados por el dinamismo y complejidad de las tecno-interacciones que realizan los emisores y receptores de la información y el conocimiento.

3. La acción de las organizaciones de conocimiento: su naturaleza, alcance, procesos, medios y tecnologías, constituyen el objeto de estudio de las ciencias de la información y la comunicación, porque son instituciones articuladas en función de la comunicabilidad; entendidas desde la mediación tecnológica, socio-cultural y cognoscitiva,

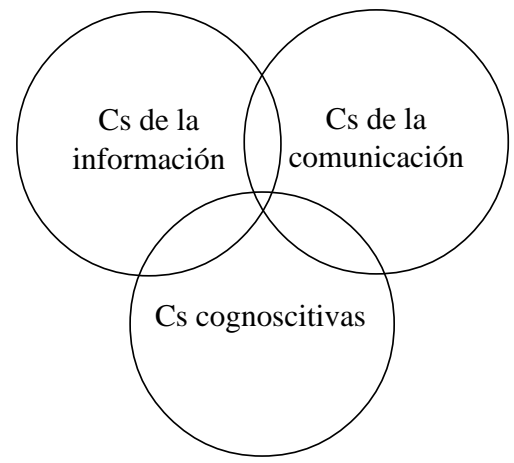


MALLA TEÓRICA

(REPRESENTACIÓN DE LA LÍNEA TEÓRICA INTEGRADA)

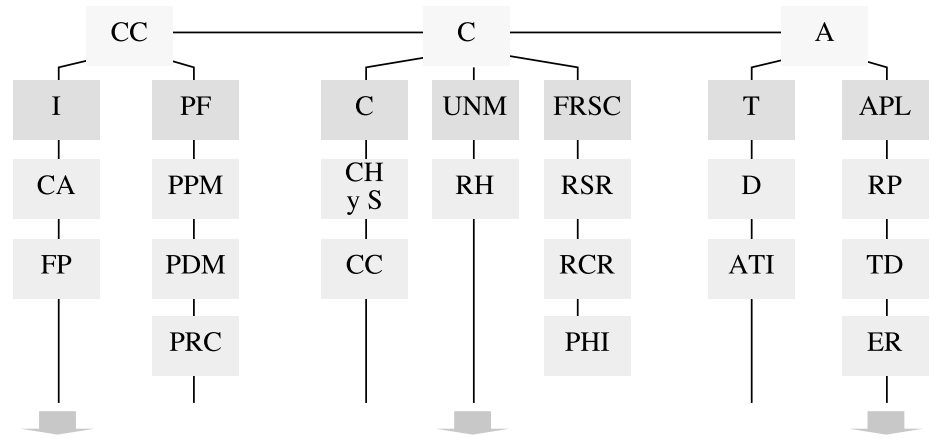

Plano de observación: documentos internacionales, nacionales y regionales

Cuadro explicativo de conceptos que conforman la línea teórica integrada

- Conceptos teóricos

1. Comunicación-mediación del conocimiento $(\mathrm{CC})$

2. Cibersociedad (C)

3. Aprendizaje (A)

- Conceptos derivados (Comunicaciónmediación del conocimiento)

1. Infraestructura (I)

2. Procesos y productos facilitadores $(\mathrm{PF})$

- Conceptos derivados (Cibersociedad)

1. Concepciones (C)

2. Uso de nuevos medios (UNM)

3. Nuevas formas de relación social y cognoscitiva (NFRSC)

- Conceptos derivados (Aprendizaje)

1. Tipos de aprendizaje $(\mathrm{T})$

3. Aplicación del aprendizaje (APL)

- Conceptos empíricos (Infraestructura)

1. Concepción de la acción de las organizaciones de conocimiento (CA)

2. Formación de personal (FP)

- Conceptos empíricos (Procesos y productos facilitadores)

1. Procesos previos a la mediación cognoscitiva (PPM)
2. Procesos y productos de mediación cognoscitiva (PDM)

3. Procesos de recepción cognoscitiva (PRC)

- Conceptos empíricos (Concepciones)

Concepción de hombre y sociedad (CHS)

Concepción de conocimiento (CC)

- Conceptos empíricos (Uso de nuevos medios)

Uso de recursos hipermedia (URH)

- Conceptos empíricos (Nuevas formas de relación social y cognoscitiva)

Relación social reticular (RSR)

Relación cognoscitiva reticular (RCR)

Procesamiento holístico de la información (PHI)

- Conceptos empíricos (Tipos de aprendizaje)

Deuteroaprendizaje (D)

Aprendizaje tecnológico-informativo (ATI)

- Conceptos empíricos (Aplicación del aprendizaje)

1. Resolución de problemas (RP)

2. Toma de decisiones (TD)

3. Emprender retos (ER)

Sociedad del conocimiento, la comunicación y el aprendizaje 
pues esta perspectiva es mucho más acorde con lo que se espera en estos momentos de las organizaciones de conocimiento, es decir, producir en los usuarios los aprendizajes tecnológico-informativos permanentes para contribuir con su inserción activa en la sociedad del conocimiento, la comunicación y el aprendizaje.

\section{BIBLIOGRAFÍA}

Agudo, A. (2001) "Ética en la sociedad de la información. Reflexiones desde América Latina y el Caribe (I Parte)", en INFOLAC. Boletín trimestral del Programa Sociedad de la Información para América Latina y el Caribe. Vol. 14. No 3. Octubre-diciembre. Quito-Ecuador. pp.19-23.

BAGOZZI, R. P. y Phillips, L. (1982) "Representing and testing organizational theories: A holistic construal". Administrative Scieence Quartely. 27: 459489.

Beckett, C. et al. (2000) "Desconstruyendo la identidad del homo-digitalis", en Revista Comunicación, No. 109, Caracas-Venezuela: Centro Gumilla, pp. 5863.

CASTELLS, M. (1999) La era de la información: economía, sociedad y cultura. Madrid-España: Siglo XXI.

CARTIER, M. (1992) "Un nuevo modelo de acceso al conocimiento", en Silvio, J. (Comp). Calidad, tecnología y globalización en la educación superior latinoamericana, y en INFOLAC, No. 5 (3-4) pp. 3-19. Caracas-Venezuela: Edic. CRESALC-UNESCO.

CRONIN, B. (1983) "Post-industrial society: some manpower signs for the library/ information", en Journal of information science, 7: 1-14.

CLOUTIER, J. (1992) "EMEREC, La comunicación audio-scripto-visual y la telemediática", en Silvio, J. (Comp.). Calidad, tecnología y globalización en la educación superior Latinoamericana. Caracas-Venezuela: Edic. CRESALCUNESCO.

DRUCKER, P. (1993) La sociedad postcapitalista. Bogotá-Colombia: Editorial Norma.

GALINDO, L. J. (1998) Técnicas de investigación en sociedad, cultura y comunicación. Pearson: Pearson.

GARCíA, D. (1992) Aspectos teóricos y organizacionales del proceso de comunicación en unidades documentales. Trabajo de ascenso (no publicado) para ascender a la categoría de profesora titular. Maracaibo-Venezuela: Universidad del Zulia, Escuela de Bibliotecología y Archivología.

HEMPEL, C. G. (1988) Fundamentos de la formación de conceptos en ciencia empírica. Madrid-España: Edit Alianza.

MARTín Barbero, J. (1998) De los medios a las mediaciones. Comunicación, cultura y hegemonía. Bogotá-Colombia: Edic. Convenio "Andrés Bello". 
MARTín Serrano, M. (1997) "La mediación de los medios”, en Barbero y Silva (Comp). Proyectar la comunicación. Bogotá- Colombia: Tercer Mundo Editores. pp. 137-156.

MithEN, S. (1998) Arqueología de la mente. Orígenes del arte, de la religión y de la ciencia. Barcelona-España: Edit. Crítica.

MoORE, C.; Hajnal, P.; Manning, C. (1997) "Los servicios de información a través del mundo. A Bibliotecas y servicios de información. (Canadá y Estados Unidos)", en UNESCO. Informe mundial sobre la información. Madrid España. pp.104-113.

MoriLlo, N. (2000) Resumen del diagnóstico y recomendaciones de la Biblioteca Pública Central “María Calcaño”. Gobernación del Estado Zulia. Mimeografiado. Maracaibo-Venezuela.

MORALES, E. (1997) "Los servicios de información a través del mundo. Las bibliotecas y servicios de información. (América Latina y el Caribe)", en UNESCO. Informe mundial sobre la información. Madrid-España. pp. 114129.

NIÑO, G. (1994) "Evaluación de los depósitos documentales públicos de la ciudad de Maracaibo". Ponencia presentada en el V Seminario sobre Patrimonio Histórico, Cultural y Artístico. "Memoria documental archivística del Zulia”, Maracaibo 24 y 25 de noviembre.

NONAKA, I. y Takeuchi (1998) La organización creadora de conocimiento. Oxford: Oxford University Press. U.K

ORTEGA et al. (1999) "Proyecto de Especialización en Archivos Públicos y Privados". Documento de trabajo para organizar los estudios de posgrado en la Escuela de Bibliotecología y Archivología de LUZ. Mimeografiado. Maracaibo-Venezuela: Universidad del Zulia.

ORTIZ, R. (2002) entrevista en Portal de Comunicación. Por: Rodrigo Gómez (disponible en línea) http://www.innovarium.com/ConferenciasXXI/ Entrevista\%20Renato\% 20Ortiz.pdf. (consulta: 21-12-03).

OTLET, P. (1934) Traité de Documentation. Le livre sur le livre. Théorie et practique. Bruselas-Bélgica: Edit. Mundaneum.

PÁEZ, I. (1992) Gestión de la inteligencia, aprendizaje tecnológico y modernización del trabajo informacional. Retos y oportunidades. Instituto de Estudios del Conocimiento. Caracas-Venezuela: Universidad "Simón Bolívar".

PASQUALI, A. (2003) “ ¿Sociedad de la información o derecho a la comunicación?". Diario El Nacional, 28 de diciembre, A-6. Caracas-Venezuela.

PINEDA, M. et al. (2001) De los mass media a los medios telemáticos: teorías y modelos. Maracaibo-Venezuela: CONDES-Universidad del Zulia.

- (2000) "Los paradigmas de la comunicación: nuevos enfoques teórico-metodológicos", en Diálogos de la comunicación. Nos. 59-60. FELAFACS. Lima-Perú. pp. 265-272. 
PRIMERA, N. (1992) "Situación actual y situación deseada de la Red de Bibliotecas Públicas del Estado. 1993-1995”. Secretaría de Educación del Estado Zulia. Mimeografiado. Maracaibo-Venezuela: Red de Bibliotecas Públicas.

POPPER, K. (1973) La lógica de la investigación científica. Madrid-España: Teknos.

- (1992) Conocimiento objetivo. Un enfoque evolucionista. Madrid-España: Teknos.

SHERA, J. (1972) The foundations of education for librarianship. New York, USA: Beker and Hayes.

SODRÉ, M. (2001) Entrevista con el profesor Muniz Sodré, en Revista Fronteiras. Estudios mediáticos, Vol. III No. 1 Junio 2001. Brasil: Programa de PósGraduacao em Ciencias da Comunicacao. UNISINOS.

TERCEIRO, J. (1996) La sociedad digital. Del homo sapiens al homo digitalis. Madrid-España: Alianza Editorial.

TREJO, D. R. (1999) “ Desafíos y divergencias de la sociedad de la información en América Latina” (disponible en línea) http://www.recyt.org.ar/Delarbre.htm (Consulta: 13-09-02).

VILAS, C. (1999) "Seis ideas falsas sobre la globalización. Argumentos desde América Latina para refutar una ideología” (disponible en línea) http:// www.globalizacion.org/biblioteca/Vilas\%20Globalizacion\%20Falsa.htm (Consulta: 15-02-03).

VITIELLO, G. (1997) "Los servicios de información a través del mundo. Las Bibliotecas y servicios de información. (Europa Occidental)", en UNESCO. Informe mundial sobre la información. Madrid- España. pp. 88-103. 